\title{
Numerical Approach to Determining Windings' Thermal Conductivity
}

\author{
Amal Zeaiter, Student Member, IEEE, Matthieu Fénot, Didier Saury
}

\begin{abstract}
This study concerns the characterization of windings' thermal conductivity, which is of primordial importance for thermal management of a high power density electrical machine. Indeed, such machines exhibit high heat dissipation in the windings' area. A precise knowledge of this conductivity value is therefore important for good thermal predictions. Unfortunately, the heterogeneity of the windings and the heat production repartition make it difficult to calculate easily winding's equivalent thermal conductivity. The influence of four thermal and geometrical parameters on the windings' equivalent thermal conductivity is numerically studied on a Finite Element Analysis software, allowing determining more precisely this characteristic. The impregnation's thermal conductivity, the conductors' number, the fill factor and the configuration of the copper wires inside the slot are investigated. The equivalent thermal conductivity, principally controlled by the impregnation thermal conductivity, the slot fill factor and the conductors' disposition, is expressed in terms of the first two parameters in its worst-case configuration.
\end{abstract}

Index Terms--Thermal management, windings, thermal conductivity, Finite Element Modeling (FEM)

\section{INTRODUCTION}

$\mathrm{E}$ LECTRIC Machines are nowadays essential power sources for many different applications in multiple domains and sectors, and the interest of using the electrical power is increasing rapidly with time, especially with the development of a new generation of high power machines. This is particularly true for motors designed for transport propulsion (vehicles, boats, and even airplanes in an advanced future time [1], [2]) mainly because of the compactness of such motors. Due to their high power densities, different specific cooling solutions are currently studied [3]-[6]. Therefore, during the sizing procedure and before designing the adequate cooling system, it is particularly important to determine precisely their thermal characteristics to predict their temperatures. In particular, windings' thermal model could have a huge influence on the predicted temperature. For example, based on a sensitivity study conducted on a developed numerical thermal model of a 0.9 MW high-power density machine of permanent magnet synchronous type, the equivalent conductivity of the winding in both radial and ortho-radial (or angular) directions appeared to relatively influence the temperature rise in the slots and at the end-windings. It reaches in some cases 15 degrees for $10 \%$ of variation in this property's value. This is due to the high resistance of the windings' assembly acting as an inhibitor of the heat evacuation from the windings to the outside through the stator laminations and the frame. Winding is a heterogeneous region in the machine, generally constituted of copper wires, called the conductors, and an insulation material (generally the epoxy resin for impregnation and the varnish covering the conductors). For global thermal models, heterogeneous regions are generally represented by a global equivalent thermal conductivity that characterizes the heat transfer phenomenon from such critical hot areas inside the machine to a non-relative connected medium. It is common to use provided empirical correlations that consider only the materials' percentages and conductivities, and are careless of the conductors' disposition and spacing [7]-[9]. Authors generally assimilate coils to heterogeneous materials consisting of wires or fibers enclosed in an impregnation material. In fact, in such assumption, the distribution of heat dissipation is not considered, and it is assumed that the heat is produced at the center point of the body, while in fact the heat production is pertained only to the windings' conductors, generally dispersed within the entire slot. From a thermal viewpoint, a centered dissipation of heat induces a thermal resistance twice that of the uniformly distributed case. This will also come out with twice the temperature difference from the center to the wall surface. Moreover, geometries are generally limited to some generic conductors' configuration. There are some authors who investigated the windings' equivalent thermal conductivities or contributed to the modeling of windings' in a realistic approximation [10], [11]. Other works provided analytical correlations or methods for windings made of Litz wires [12], [13]. In the former, the authors determined the thermal conductivity of Litz wires' windings in terms of different thermal and geometrical characteristics of the slot composition, based on the analogy between electrostatics and heat transfer. They compared their results to that of solid wires using the empirical Hashin and Shtrikman approximation [9]. Whereas, in the latter, the researchers validated their methods with experimental data of Litz wire windings from [14] who conducted series of numerical and experimental tests allowing to find out equivalent thermal parameters. [15], [16] worked in this field to evaluate numerically the equivalent thermal conductivity for a representative windings' thermal configuration with heat dissipation in conductors. They investigated the effect of different parameters (the

This project has received funding from the Clean Sky 2 Joint Undertaking under the European Union's Horizon 2020 research and innovation program under grant agreement $\mathrm{N}^{\circ} 715483$.

A. Zeaiter, M. Fénot and D. Saury are with the Department of Fluids, Thermal and Combustion Sciences - Pprime Institute UPR 3346, Poitiers, 86000 France (e-mails: amal.zeaiter@ensma.fr, matthieu.fenot@ensma.fr, didier.saury@ensma.fr). 
fill factor, the disposition of the winding inside the slot and the conductors' number) but only for a specific circular crosssection of the conductors. In the present study, the heat transfer inside windings of rectangular-shaped conductors and slot sections, of a high-power density motor, is studied numerically using numerical Finite Element (FE). The aim of this study is to analyze the thermal behavior of the slot windings to determine numerically their radial and ortho-radial thermal conductivities. Rectangular copper conductors, epoxy resin impregnation and polyimide film insulation are modeled. As set forth, the heat is produced singularly and proportionally in each conductor. Four main parameters interest this work: the thermal conductivity of the impregnation, the disposition of the conductors in the stator slot, the slot fill factor and the conductors' number. The study was carried out to evaluate the importance of each parameter in temperature rise and determine the thermal conductivity of the whole winding.

\section{ANALYTICAL DESCRIPTION}

Generally, motors' heat transfer models are resolved based on Lumped Parameter Thermal Model (LPTM) method where a temperature and a heat source are associated to each node; nodes are connected one to the other with thermal resistances and the local nodal network of the stator slot is shown in Fig. 1.

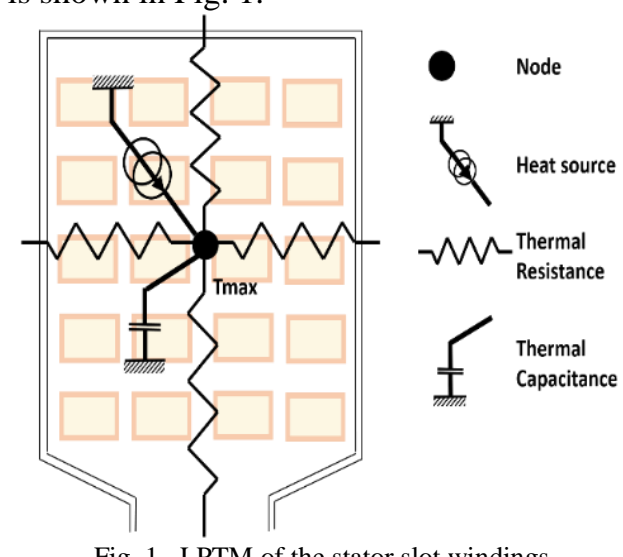

Fig. 1. LPTM of the stator slot windings.

For the winding model, slots are generally represented with a rectangular section. Therefore, radial and angular conductances ( $G_{\text {cond-rad }}, G_{\text {cond-ang }}$ respectively) are mono-dimensional, expressed in Cartesian coordinate system. They are correlated to the directional thermal conductivities as indicated in (1) and calculated from the center of the slot generally considered as the hottest point of the winding for nodal network analysis.

$$
\left\{\begin{array}{l}
G_{\text {cond-rad }}=\frac{\lambda_{e q}^{r} s_{r}}{e_{r}} \\
G_{\text {cond-ang }}=\frac{\lambda_{e q}^{a} s_{a}}{e_{a}}
\end{array}\right.
$$

Where $\mathrm{S}$ is the slot radial section, $e_{r}$ is the distance from the slot center to the slot upper surface (equal to half the slot height), and $e_{a}$ is the distance from the slot center to the slot lateral side (equal to half the slot width). Fig. 2 depicts the slot geometry dimensions: $S_{a}=L \times 1, S_{r}=l \times 1, e_{r}=L / 2, e_{a}=l / 2$, as the depth is set equal to $1 \mathrm{~m}$.

The directional thermal conductivities are generally estimated based on the copper wires' disposition and the conductivities and resin conductivity only; and the total heat production is supposed to be concentrated at the center of the slot, which tends to overestimate the maximal temperature. Our goal is to calculate directional thermal conductivities considering a distributed heat production to predict more accurately the maximal temperature of the winding.

\section{A. FE solving on ABAQUS}

The study consists in conceiving the windings' model on ABAQUS software, for which the aforementioned parameters (the thermal conductivity of the impregnation material, the disposition of the conductors in the stator slot and the slot fill factor) are set according to machine design characteristics. A study was carried out by varying the element base size and consequently their number to be sure that results are independent of the meshing.

The radial thermal conductivity of the winding reference model is determined by setting a boundary temperature at the upper side and considering adiabatic conditions simultaneously at both lateral sides (teeth sides) and at the lower surface (airgap side). Thermal loads are imposed uniformly distributed in the conductors' part. With these assumptions, the heat flux is forced to take the radial path and the resulting maximal temperature is extracted from the simulation. Using the heat production $\dot{Q}, G_{\text {cond }}$ is calculated according to equations (2) and (3): 


$$
\begin{aligned}
& 0=G_{\text {cond }}\left(T_{w}-T_{\max }\right)+\dot{Q} \\
& G_{\text {cond }}=\frac{\dot{Q}}{\left(T_{\max }-T_{w}\right)}
\end{aligned}
$$

Where $G_{\text {cond }}$ is the thermal conductance in considered direction, $\dot{Q}$ is the dissipated heat flux, $T_{w}$ is the wall prefixed temperature and $T_{\max }$ is the obtained maximal winding temperature representative of the windings' temperature when resolving the lumped parameter thermal model.

It allows evaluating the thermal conductivity along the slot radial direction by equalizing the expression of the global conductance $G_{\text {cond }}$ in (3) from numerical results and analytical expressions of the conductances $G_{\text {cond-rad }}$ and $G_{\text {cond-ang }}$ in (1).

The ortho-radial conductivity is calculated similarly by changing the adiabatic zones and the temperature boundary condition. As such, the heat flows angularly, and the equivalent ortho-radial conductance, then conductivity, are determined.

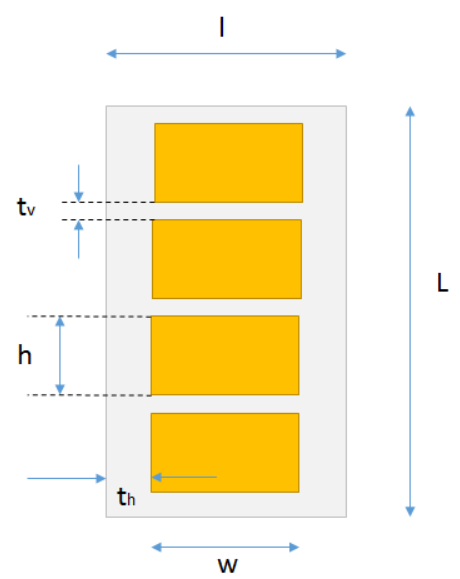

Fig. 2. Slot geometry section with four rectangular conductors in vertical configuration.

\section{B. Geometry and properties}

In the model of the winding, rectangular copper conductors of $400 \mathrm{~W} / \mathrm{m} \mathrm{K}$ thermal conductivity are impregnated with an epoxy resin material. A polyimide liner covers the external surface of the whole winding with 100um thickness. Fig. 2 shows an example of the two-dimensional axial section of the winding inside the slot, and Table I groups the fixed dimensions' values, noting that parameters $\mathrm{w}$ and th will be changed only in the last configuration study. Other dimensions ranges are presented in Table II; the spacing between conductors as well as their height are denoted by one parameter each: tv and $\mathrm{h}$ respectively and they change with the variation of the fill factor, conductors' number and the conductors' disposition. The model is simulated in 2-D and the depth is used for body heat flux definition and is equal to $1 \mathrm{~m}$.

TABLE I

PRIMARILY FIXED DIMENSIONS OF THE SLOT

\begin{tabular}{l|c|c|c|c}
\hline Dimension & $\mathrm{L}$ & 1 & $\mathrm{w}$ & $\mathrm{t}_{\mathrm{h}}$ \\
\hline Value (mm) & 21.28 & 10.24 & 7.24 & 1.5 \\
\hline
\end{tabular}

TABLE II

VARIABLE DIMENSIONS' RANGES FOR THE VARIATIONS OF CONDUCTORS' NUMBER AND THE FILL FACTOR

\begin{tabular}{l|c|c}
\hline Dimension & $\mathrm{h}$ & $\mathrm{t}_{\mathrm{v}}$ \\
\hline Min (mm) & 0.94 & 1.13 \\
\hline Max (mm) & 15.05 & 14.36 \\
\hline
\end{tabular}




\section{RESULTS AND ANALYSIS}

\section{A. Impregnation Thermal Conductivity}

Multiple types of epoxy resin can be used for winding impregnation. They are characterized by their thermal conductivities, which should be considered in thermal studies as well as in machine designs. In the current work, initial numerical tests are carried out with resins of different thermal conductivities $\lambda_{i m p}: 0.3,0.5,0.8$ and $1.8 \mathrm{~W} / \mathrm{m} \mathrm{K}$. Then for each resin, the other parameters of the study are evaluated.

As can be seen in Figure 3, an increase of the resin thermal conductivity causes a nearly proportional increase in thermal conductivity of the whole slot (at least up to $0.8 \mathrm{~W} / \mathrm{m} \mathrm{K}$ ) inducing a stronger heat evacuation process from the main heat source of the motor. This parameter influences positively the windings' thermal behavior in both radial and angular directions relatively more in the former.

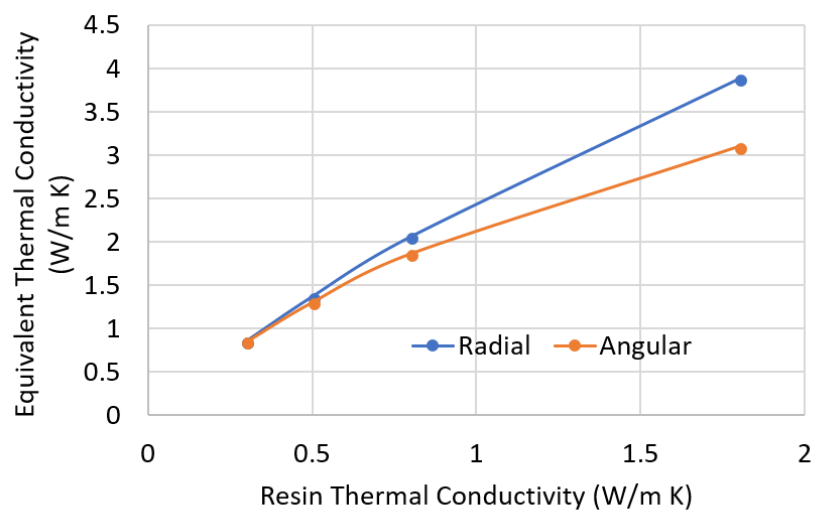

Fig. 3. Windings' equivalent thermal conductivity vs. resin thermal conductivity in radial and angular directions.

\section{B. Number of Conductors}

In electric motors, the number of conductors in the slot will contribute to determining the Joule losses. Besides being main actors in the temperature rise, these losses are not the only aspects of the thermal influence of conductors' number. In the purpose of thermal conductivity determination, and always with a 0.5 of filling factor, their number will be varied from one to 16 conductors and the variation of the equivalent thermal conductivity is plotted for each resin thermal conductivity previously defined in Fig. 4 ( $a$ and $b$ ).

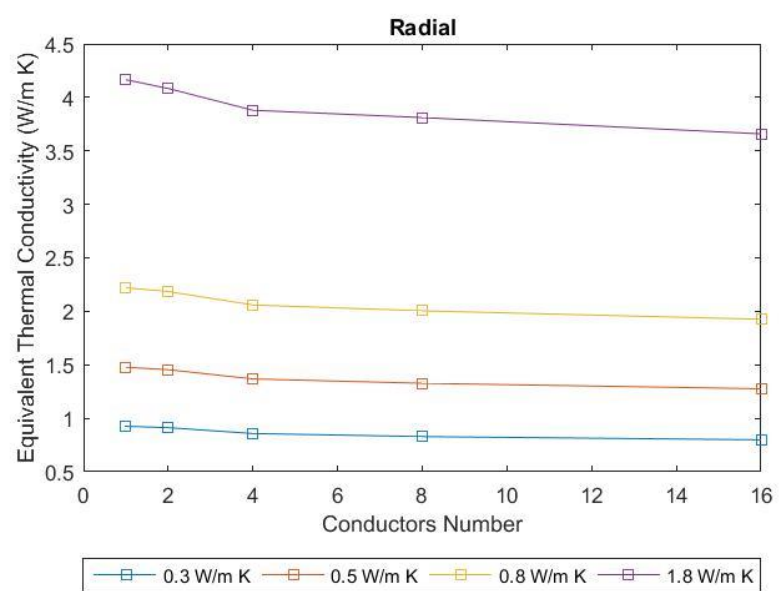

(a)

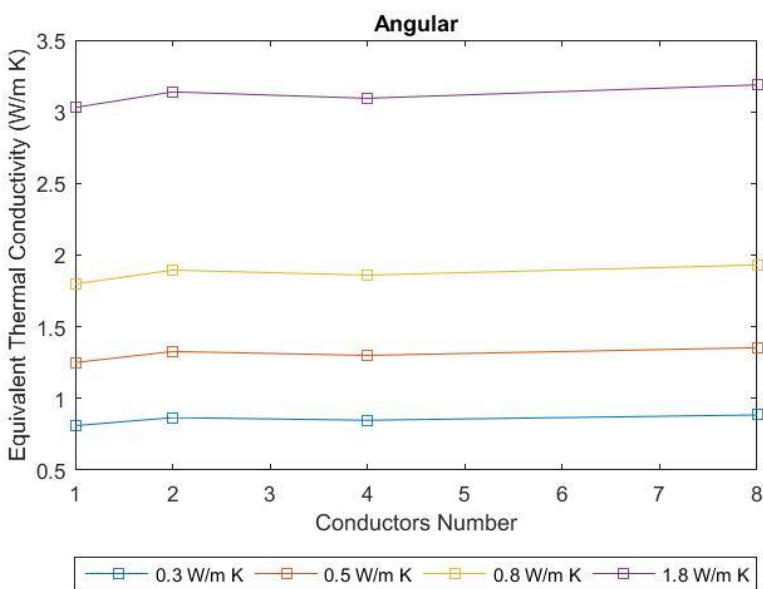

(b)

Fig. 4. Windings' equivalent thermal conductivity as function of the number of conductors for different impregnation material thermal conductivities in (a) radial (b) angular directions.

The results shown in both radial and ortho-radial directions prove that changing the number of conductors while maintaining the same copper section inside the slot will slightly influence the thermal conductivity since the relative variation is less than $7 \%$ in both directions. Radially, the conductivity is slightly improved when reducing the conductors' number since the resin layer is thinner. For one conductor the thickness between $T_{\max }$ zone and the slot upper wall is smaller and equal to 1 
time $\mathrm{t}_{\mathrm{v}}$ in this case $3.12 \mathrm{~mm}$, while for 16 conductors the total thickness is equal to $5.87 \mathrm{~mm}$ (16 times the resin layer thickness). Practically, this parameter can be neglected in the determination of the equivalent thermal property in a vertical disposition.

\section{Fill Factor}

The fill factor $\tau$ is defined by the ratio (or percentage) of the copper conductors to the impregnation. Slots with fill factors of 0.2 and up to 0.6 are compared from a thermal viewpoint. The study has been conducted on two configurations of the slot: with four and with two conductors.

Graphs of Fig. 5 (a and b) represent the evolution of the windings' directional thermal conductivities in terms of the fill factor.

Results for both (2 and 4-conductors) slot types, respectively in dashed and solid lines, are globally consistent with a comparable percentage of variation. These results show that the heat dissipation process inside the slot relies considerably on this parameter. In the radial direction, the effect of the fill factor is significantly obvious with an average increase going from $23 \%$ up to around $65 \%$ in thermal conductivity when switching from 0.2 to 0.3 and from 0.5 to 0.6 of fill factor. This is explained by the reduction of the impregnation layers' thicknesses in vertical (radial) direction. Despite the important increase in radial thermal conductivity in terms of the fill factor, it is evident that the influence of this parameter turns out to be less obvious when increasing the impregnation material's thermal conductivity getting closer to the conductors.

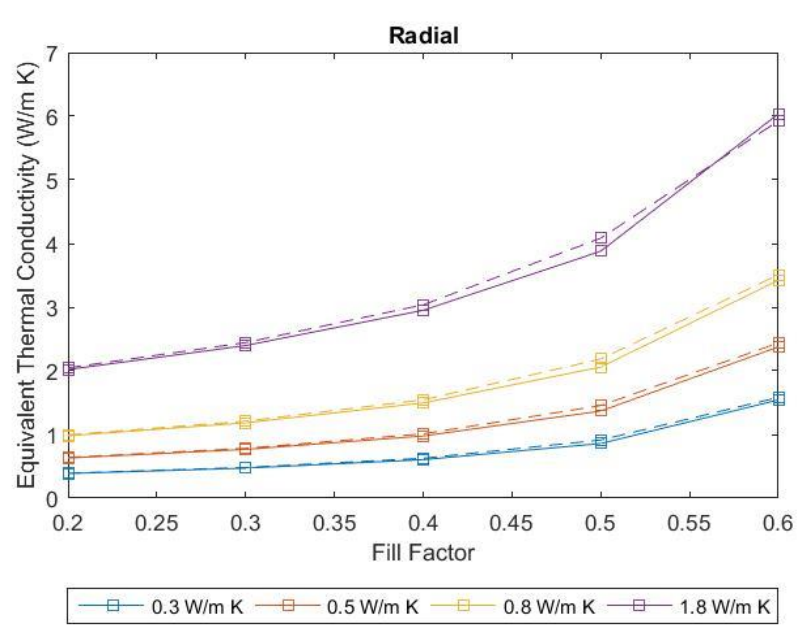

(a)

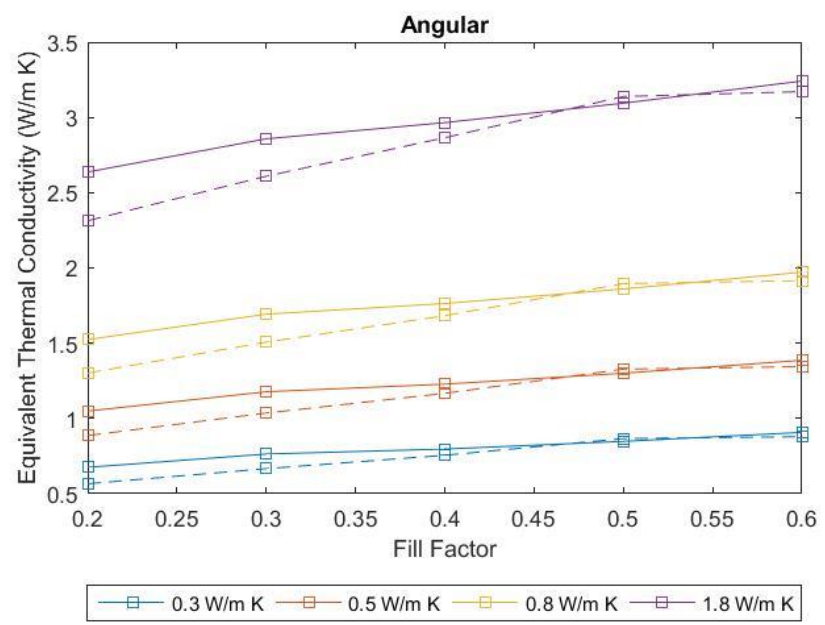

(b)

Fig. 5. Windings' equivalent thermal conductivity as function of impregnation fill factor for different impregnation material thermal conductivities in (a) radial (b) angular directions for four conductors' slot (solid lines) and 2 conductors' slot (dashed lines).

\section{Conductors' Disposition}

In this part, two conductors' dispositions are investigated, and results are compared to those of vertical disposition with four rectangular copper conductors. The first one is the squared configuration shown in Figure 6a, while the second is geometrical disposition (horizontal configuration) is presented in Figure 6b. Dimensions are grouped in Table III for each configuration. The simulation considers the same conditions as for the vertical case.

The results of the simulations are depicted in Figure 7. It is evident according to the graphs that the squared configuration is more advantageous in the angular direction. 


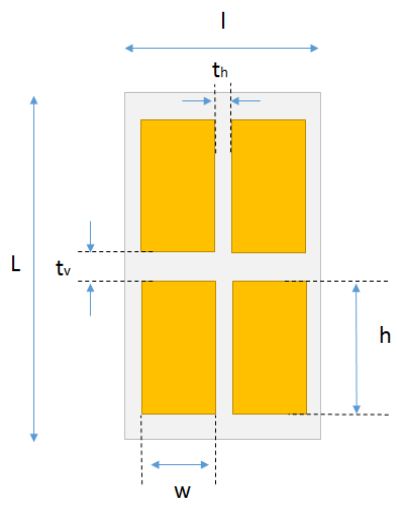

(a)

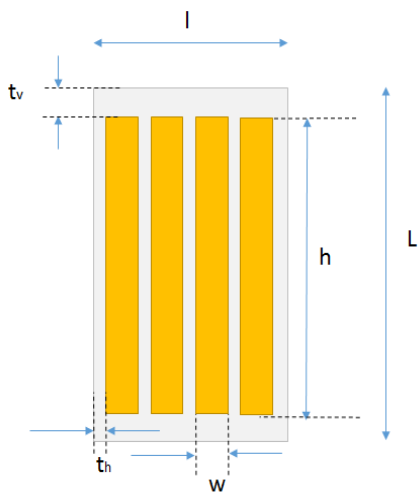

(b)

Fig. 6. Slot geometry section with four rectangular conductors in (a) squared (b) horizontal configurations.

TABLE III

DIMENSIONS CORRESPONDING TO CONFIGURATIONS (A) SQUARED AND (B) HORIZONTAL

\begin{tabular}{l|c|c|c|c}
\hline Dimension & $\mathrm{h}$ & $\mathrm{w}$ & $\mathrm{t}_{\mathrm{v}}$ & $\mathrm{t}_{\mathrm{h}}$ \\
\hline Value (mm) & 8 & 3.41 & 1.76 & 1.144 \\
\hline
\end{tabular}

(a)

\begin{tabular}{l|c|c|c|c}
\hline Dimension & $\mathrm{h}$ & $\mathrm{w}$ & $\mathrm{t}_{\mathrm{v}}$ & $\mathrm{t}_{\mathrm{h}}$ \\
\hline Value (mm) & 18.28 & 1.49 & 1.5 & 0.856 \\
\hline
\end{tabular}

(b)

Compared to the horizontal configuration this is due to the double impregnation layers at each side of the slot forming a greater total thickness than in squared configuration (1.5 times).

In vertical disposition, the average gain between vertical and squared configuration is around $23 \%$ in the radial direction and $26 \%$ in the angular one. This is justified by the fact that the impregnation thickness at the lateral sides is $27 \%$ smaller in the squared configuration, which acts on further heat dissipation and is evident in the angular thermal conductivity results. Horizontal disposition is thermally more beneficial in the radial direction. It is justified first by the thinner impregnation layer but mostly by the greater heat path length in the copper from the bottom to the top slot surface $(18.28 \mathrm{~mm})$.

The radial conductivity variation is more complex in the vertical disposition; the heat dissipated in the lower conductor must cross four impregnation layers of $4.544 \mathrm{~mm}$ thickness, whereas in the square disposition the thickness is reduced to $3.52 \mathrm{~mm}$.

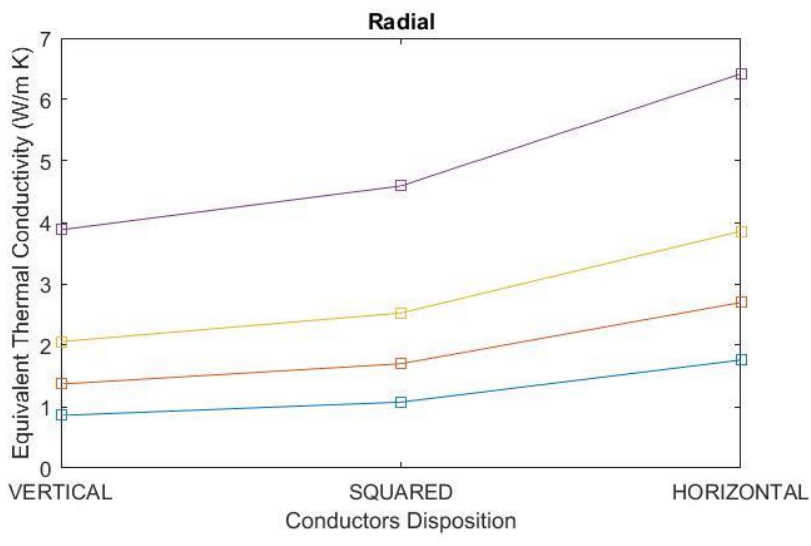

$\square-0.3 \mathrm{~W} / \mathrm{m} \mathrm{K} \square-0.5 \mathrm{~W} / \mathrm{m} \mathrm{K} \square-0.8 \mathrm{~W} / \mathrm{m} \mathrm{K} \square-1.8 \mathrm{~W} / \mathrm{m} \mathrm{K}$

(a)

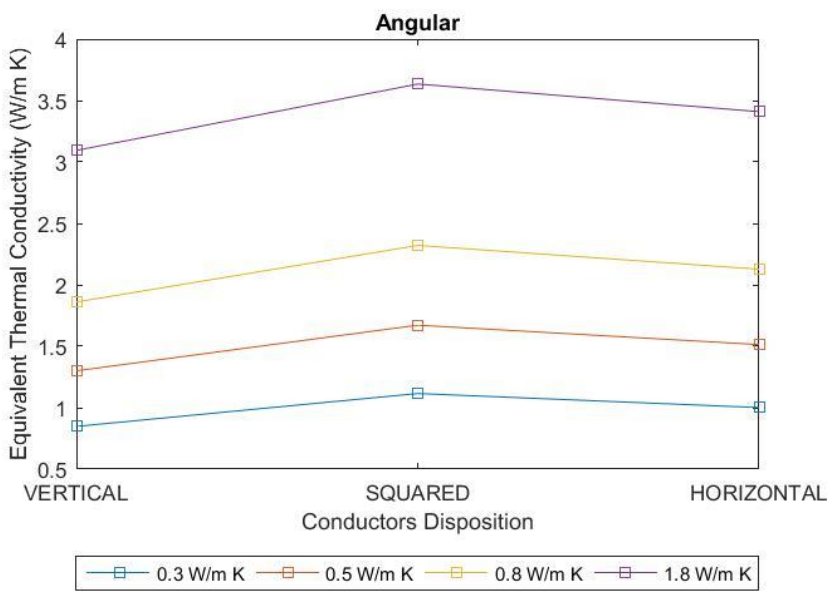

(b)

Fig. 7. Windings' equivalent thermal conductivity as function of the configuration for different impregnation material thermal conductivities in (a) radial (b) angular directions. 


\section{E. Thermal Conductivity Expression}

Based on the data obtained and considering only the most influencing parameters which are the fill factor $\tau$ and the impregnation thermal conductivity $\lambda_{\text {imp }}$, a general expression of the thermal conductivity was elaborated and can be used for the slot and end-windings thermal resistances' calculation. These resistances are part of the thermal nodal network allowing to numerically predict temperature in the motor and essential to the design of reliable high power and super high power electric machines. The obtained expression (4) is a second order polynomial correlation whose constants depend on the cylindrical directions (radial and angular). Table IV groups the values of these constants.

$$
\lambda_{e q}=A+B \lambda_{i m p}+C \tau+D \tau \lambda_{i m p}+E \tau^{2}
$$

TABLE IV

CONSTANTS OF THE EXPRESSION OF EQUIVALENT THERMAL CONDUCTIVITY

\begin{tabular}{l|c|c|c|c|c}
\hline & A & B & C & D & E \\
\hline Radial & 2.05 & 0 & -12.14 & 4.39 & 17.4 \\
\hline Angular & 0.23 & 1.17 & 0.94 & 0.56 & -0.57 \\
\hline
\end{tabular}

The obtained correlation is compared to Hashin and Shtrikman approximation (5) ( $\lambda_{c u}$ is the copper thermal conductivity) in an adapted configuration, i.e. considering the insulator film. This comparison confirms that there is a difference in the results that can be significantly important in some cases. For instance, the equivalent thermal conductivities in angular direction computed from the resulting correlation for a fill factor of 0.3 , are relatively higher than those calculated using classical approximation, with an average of $22 \%$. Fig. 8 shows the disparity in values.

$$
\lambda_{e q}=\lambda_{i m p} \frac{(1+\tau) \cdot \lambda_{c u}+(1-\tau) \cdot \lambda_{i m p}}{(1-\tau) \cdot \lambda_{c u}+(1+\tau) \cdot \lambda_{i m p}}
$$

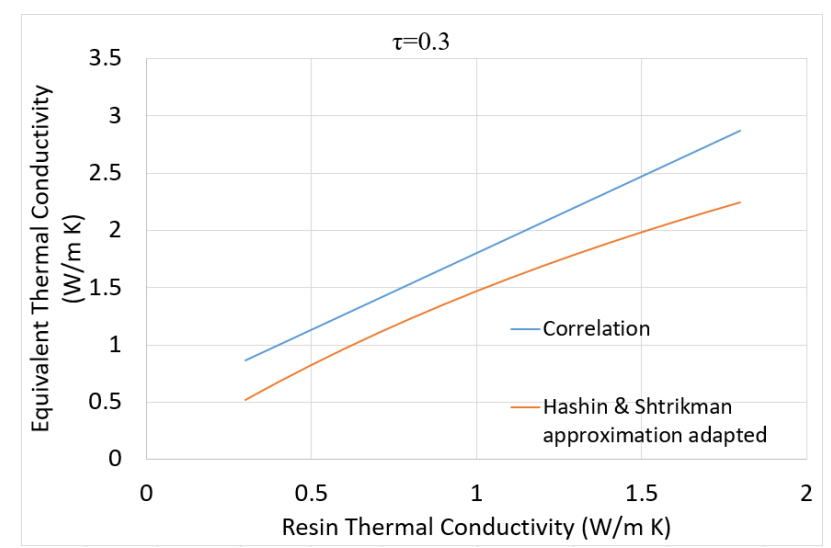

Fig. 8. Windings' equivalent angular thermal conductivity as function of resin thermal conductivity for 0.3 fill factor.

\section{CONCLUSION}

In the design modeling of windings' thermal conductivity inside the slot of a high power electrical machine, it is important to consider the disposition of the conductors, but most importantly the impregnation thermal conductivity and the fill factor. The latter two parameters influence the equivalent thermal conductivity and determine its value. They basically determine the number and the thickness of the impregnation's layers contributing to the temperature rise in this critical zone. The number of conductors has a less significant effect on the overall thermal behavior of the winding. Modeling accurately the high heat densities' evacuation from such electric machines, strongly depends on the windings' thermal conductivity calculation. This study provides correlations to compute this thermal property, showing a difference in values compared to other classical correlations. 


\section{REFERENCES}

[1] M. Bogomolov, "Concept study of $20 \mathrm{MW}$ high-speed permanent magnet synchronous motor for marine propulsion," 2013. [Online]. Available: http://repository.tue.n1/836d0d0d-4f68-499e-97c7-0cb3fa2d0a00. [Accessed: 19-Feb-2018].

[2] Siemens, "Electric propulsion components with high power densities for aviation," presented at the Transformative Vertical Flight Workshop, 08-Mar2015.

[3] T. Davin, J. Pellé, S. Harmand, and R. Yu, “Experimental study of oil cooling systems for electric motors,” Applied Thermal Engineering, vol. 75, pp. 1-13, Jan. 2015.

[4] P. Lindh et al., "Direct Liquid Cooling Method Verified With an Axial-Flux Permanent-Magnet Traction Machine Prototype," IEEE Transactions on Industrial Electronics, vol. 64, no. 8, pp. 6086-6095, Aug. 2017.

[5] M. Liu, Y. Li, H. Ding, and B. Sarlioglu, "Thermal management and cooling of windings in electrical machines for electric vehicle and traction application," in 2017 IEEE Transportation Electrification Conference and Expo (ITEC), 2017, pp. 668-673.

[6] A. Tüysüz, F. Meyer, M. Steichen, C. Zwyssig, and J. W. Kolar, "Advanced Cooling Methods for High-Speed Electrical Machines," IEEE Transactions on Industry Applications, vol. 53, no. 3, pp. 2077-2087, May 2017.

[7] General Electric, Heat transfer and fluid flow data book. 1981

[8] W. M. Rohsenow, J. P. Hartnett, and Y. I. Cho, Handbook of Heat Transfer. McGraw-Hill Education, 1998.

[9] Z. Hashin and S. Shtrikman, "A variational approach to the theory of the elastic behaviour of multiphase materials," Journal of the Mechanics and Physics of Solids, vol. 11, no. 2, pp. 127-140, Mar. 1963.

[10] D. Staton, S. Pickering, and D. Lampard, "Recent Advancement in the Thermal Design of Electric Motors." 2001.

[11] S. Ayat, R. Wrobel, J. Goss, and D. Drury, "Experimental calibration in thermal analysis of PM electrical machines," in 2016 IEEE Energy Conversion Congress and Exposition (ECCE), 2016, pp. 1-8.

[12] M. Jaritz, A. Hillers, and J. Biela, "General Analytical Model for the Thermal Resistance of Windings Made of Solid or Litz Wire," IEEE Transactions on Power Electronics, pp. 1-1, 2018.

[13] P. Romanazzi, M. Bruna, and D. A. Howey, "Thermal Homogenization of Electrical Machine Windings Applying the Multiple-Scales Method," J. Heat Transfer, vol. 139, no. 1, pp. 012101-012101-8, Aug. 2016.

[14] N. Simpson, R. Wrobel, and P. H. Mellor, "Estimation of Equivalent Thermal Parameters of Impregnated Electrical Windings," IEEE Transactions on Industry Applications, vol. 49, no. 6, pp. 2505-2515, Nov. 2013.

[15] B. Renard, "Etude expérimentale et modélisation du comportement thermique d'une machine électrique multi-fonctions : application à un alternodémarreur intégré," Poitiers, 2003.

[16] É. Chauveau, "Contribution au calcul éléctromagnétique et thermique des machines électriques application à l'étude de l'influence des harmoniques sur l'échauffement des moteurs asynchrones," Université de Nantes, 2001.

\section{BIOGRAPHIES}

Amal Zeaiter graduated from Lebanese University, Faculty of Engineering, Beirut, Lebanon. She received her M.Eng. and M.Res degrees in mechanics in 2015. She is currently pursuing her Ph.D. thesis in thermal sciences at Pprime Institute in the National Higher School of Mechanics and Aero-Engineering (ISAE-ENSMA), Poitiers, France.

Her special field of interest is the thermal modeling and cooling of an electric motor for hybrid aircraft propulsion.

Matthieu Fénot graduated from ENSMA in fluid mechanics and heat transfer in 2001. He received his Ph.D. degree in Energy and Heat Transfer in 2004.

He is currently an Assistant Professor. His fields of interest include convective heat transfer and electric motor cooling.

Didier Saury received his Ph.D. degree in Mechanics and Energy in 2003. He is currently a professor. His fields of interest include natural convection and thermal properties' measurement.

\section{ACKNOWLEDGEMENTS}

This project has received funding from the Clean Sky 2 Joint Undertaking under the European Union's Horizon 2020 research and innovation program under grant agreement No 715483 . 